\title{
A Fixed Point Approach to the Stability of Quintic and Sextic Functional Equations in Quasi- $\beta$-Normed Spaces
}

\author{
Tian Zhou Xu, ${ }^{1}$ John Michael Rassias, ${ }^{2}$ \\ Matina John Rassias, ${ }^{2}$ and Wan $\mathrm{Xin} \mathrm{Xu}^{3}$ \\ ${ }^{1}$ Department of Mathematics, School of Science, Beijing Institute of Technology, Beijing 100081, China \\ ${ }^{2}$ Pedagogical Department E. E., Section of Mathematics and Informatics, National and Kapodistrian \\ University of Athens, 4 Agamemnonos Street, Aghia Paraskevi, Athens 15342, Greece \\ ${ }^{3}$ School of Communication and Information Engineering, University of Electronic Science and Technology \\ of China, Chengdu 611731, China
}

Correspondence should be addressed to Tian Zhou Xu, xutianzhou@bit.edu.cn

Received 19 September 2010; Accepted 31 December 2010

Academic Editor: Vijay Gupta

Copyright $\odot 2010$ Tian Zhou Xu et al. This is an open access article distributed under the Creative Commons Attribution License, which permits unrestricted use, distribution, and reproduction in any medium, provided the original work is properly cited.

We achieve the general solution of the quintic functional equation $f(x+3 y)-5 f(x+2 y)+10 f(x+$ $y)-10 f(x)+5 f(x-y)-f(x-2 y)=120 f(y)$ and the sextic functional equation $f(x+3 y)-6 f(x+$ $2 y)+15 f(x+y)-20 f(x)+15 f(x-y)-6 f(x-2 y)+f(x-3 y)=720 f(y)$. Moreover, we prove the stability of the quintic and sextic functional equations in quasi- $\beta$-normed spaces via fixed point method.

\section{Introduction and Preliminaries}

A basic question in the theory of functional equations is as follows: when is it true that a function, which approximately satisfies a functional equation, must be close to an exact solution of the equation? If the problem accepts a unique solution, we say the equation is stable (see [1]). The first stability problem concerning group homomorphisms was raised by Ulam [2] in 1940 and affirmatively solved by Hyers [3]. The result of Hyers was generalized by Rassias [4] for approximate linear mappings by allowing the Cauchy difference operator $C D f(x, y)=f(x+y)-[f(x)+f(y)]$ to be controlled by $\epsilon\left(\|x\|^{p}+\|y\|^{p}\right)$. In 1994, a generalization of Rassias' theorem was obtained by Găvruţa [5], who replaced $\epsilon\left(\|x\|^{p}+\right.$ $\left.\|y\|^{p}\right)$ by a general control function $\varphi(x, y)$ in the spirit of Rassias' approach. The stability problems of several functional equations have been extensively investigated by a number of 
authors, and there are many interesting results concerning this problem (see, e.g., [6-30] and references therein).

In 1996, Isac and Rassias [30] were the first to provide applications of stability theory of functional equations for the proof of new fixed point theorems with applications. The stability problems of several various functional equations have been extensively investigated by a number of authors using fixed point methods (see $[8,10,17,22]$ ).

The functional equation

$$
f(x+y)+f(x-y)=2 f(x)+2 f(y)
$$

is said to be a quadratic functional equation because the quadratic function $f(x)=x^{2}$ is a solution of the functional equation (1.1). Every solution of the quadratic functional equation is said to be a quadratic mapping. A quadratic functional equation was used to characterize inner product spaces.

In 2001, Rassias [25] introduced the cubic functional equation

$$
f(x+2 y)-3 f(x+y)+3 f(x)-f(x-y)=6 f(y)
$$

and established the solution of the Ulam-Hyers stability problem for these cubic mappings. It is easy to show that the function $f(x)=x^{3}$ satisfies the functional equation (1.2), which is called a cubic functional equation and every solution of the cubic functional equation is said to be a cubic mapping. The quartic functional equation

$$
f(x+2 y)+f(x-2 y)=4 f(x+y)+4 f(x-y)+6 f(x)+24 f(y)
$$

was introduced by Rassias [26]. It is easy to show that the function $f(x)=x^{4}$ is a solution of (1.3). Every solution of the quartic functional equation is said to be a quartic mapping.

In this paper, we achieve the general solutions of the quintic functional equation

$$
f(x+3 y)-5 f(x+2 y)+10 f(x+y)-10 f(x)+5 f(x-y)-f(x-2 y)=120 f(y)
$$

and the sextic functional equation

$$
\begin{aligned}
& f(x+3 y)-6 f(x+2 y)+15 f(x+y)-20 f(x)+15 f(x-y) \\
& -6 f(x-2 y)+f(x-3 y)=720 f(y) .
\end{aligned}
$$

Moreover, we prove the stability of the quintic and sextic functional equations in quasi$\beta$-normed spaces via fixed point method, and also using Gajda's example to give two counterexamples for a singular case. Since $f(x)=x^{5}$ is a solution of (1.4), we say that it is a quintic functional equation. Similarly, $f(x)=x^{6}$ is a solution of (1.5), and we say that it is a sextic functional equation. Every solution of the quintic or sextic functional equation is said to be a quintic or sextic mapping, respectively.

For the sake of convenience, we recall some basic concepts concerning quasi- $\beta$-normed spaces (see [24]). 
Definition 1.1. Let $\beta$ be a fix real number with $0<\beta \leq 1$, and let $\mathbb{K}$ denote either $\mathbb{R}$ or $\mathbb{C}$. Let $X$ be a linear space over $\mathbb{K}$. A quasi- $\beta$-norm $\|\cdot\|$ is a real-valued function on $X$ satisfying the following:

(1) $\|x\| \geq 0$ for all $x \in X$ and $\|x\|=0$ if and only if $x=0$;

(2) $\|\lambda x\|=|\lambda|^{\beta}\|x\|$ for all $\lambda \in \mathbb{K}$ and all $x \in X$;

(3) there is a constant $K \geq 1$ such that $\|x+y\| \leq K(\|x\|+\|y\|)$ for all $x, y \in X$.

A quasi- $\beta$-normed space is a pair $(X,\|\cdot\|)$, where $\|\cdot\|$ is a quasi- $\beta$-norm on $X$. The smallest possible $K$ is called the modulus of concavity of $\|\cdot\|$. A quasi- $\beta$-Banach space is a complete quasi- $\beta$-normed space.

A quasi- $\beta$-norm $\|\cdot\|$ is called a $(\beta, p)$-norm $(0<p \leq 1)$ if

$$
\|x+y\|^{p} \leq\|x\|^{p}+\|y\|^{p}
$$

for all $x, y \in X$. In this case, a quasi- $\beta$-Banach space is called a $(\beta, p)$-Banach space. We can refer to $[7,17]$ for the concept of quasinormed spaces and $p$-Banach spaces.

Given a $p$-norm, the formula $d(x, y):=\|x-y\|^{p}$ gives us a translation invariant metric on $X$. By the Aoki-Rolewicz theorem, each quasi-norm is equivalent to some $p$-norm. Since it is much easier to work with $p$-norms than quasi-norms, henceforth we restrict our attention mainly to $p$-norms.

\section{General Solutions to Quintic and Sextic Functional Equations}

In this section, let $X$ and $Y$ be vector spaces. In the following theorem, we investigate the general solutions of the functional equation (1.4) and (1.5). Some basic facts on $n$-additive symmetric mappings can be found in [29].

Theorem 2.1. A function $f: X \rightarrow Y$ is a solution of the functional equation (1.4) if and only if $f$ is of the form $f(x)=A^{5}(x)$ for all $x \in X$, where $A^{5}(x)$ is the diagonal of the 5-additive symmetric map $A_{5}: X^{5} \rightarrow Y$.

Proof. Assume that $f$ satisfies the functional equation (1.4). Replacing $x=y=0$ in (1.4), one gets $f(0)=0$. Replacing $(x, y)$ with $(0, x)$ and $(x,-x)$ in (1.4), respectively, and adding the two resulting equations, we obtain $f(-x)=-f(x)$. Replacing $(x, y)$ with $(3 x, x)$ and $(0,2 x)$ in (1.4), respectively, and subtracting the two resulting equations, we get

$$
5 f(5 x)-14 f(4 x)+10 f(3 x)-120 f(2 x)+121 f(x)=0 .
$$

Replacing $(x, y)$ with $(2 x, x)$ in $(1.4)$, we have

$$
5 f(5 x)-25 f(4 x)+50 f(3 x)-50 f(2 x)-575 f(x)=0 .
$$

for all $x \in X$. Subtracting (2.1) and (2.2), we find

$$
11 f(4 x)-40 f(3 x)-70 f(2 x)+696 f(x)=0
$$


for all $x \in X$. Replacing $(x, y)$ with $(x, x)$ in (1.4), and multiplying the result by 11 , we obtain

$$
11 f(4 x)-55 f(3 x)+110 f(2 x)-1419 f(x)=0
$$

for all $x \in X$. Subtracting (2.3) and (2.4), one gets

$$
15 f(3 x)-180 f(2 x)+2115 f(x)=0
$$

for all $x \in X$. Replacing $(x, y)$ with $(0, x)$ in (1.4), and multiplying the result by 15 , one finds

$$
15 f(3 x)-60 f(2 x)-1725 f(x)=0
$$

for all $x \in X$. Subtracting (2.5) and (2.6), we arrive at

$$
f(2 x)=32 f(x)=2^{5} f(x)
$$

for all $x \in X$.

On the other hand, one can rewrite the functional equation (1.4) in the form

$$
f(x)-\frac{1}{10} f(x+3 y)+\frac{1}{2} f(x+2 y)-f(x+y)-\frac{1}{2} f(x-y)+\frac{1}{10} f(x-2 y)+12 f(y)=0
$$

for all $x \in X$. By [29, Theorems 3.5 and 3.6], $f$ is a generalized polynomial function of degree at most 6 , that is, $f$ is of the form

$$
f(x)=A^{5}(x)+A^{4}(x)+A^{3}(x)+A^{2}(x)+A^{1}(x)+A^{0}(x), \quad \text { for all } x \in X,
$$

where $A^{0}(x)=A^{0}$ is an arbitrary element of $Y$ and $A^{i}(x)$ is the diagonal of the $i$-additive symmetric map $A_{i}: X^{i} \rightarrow Y$ for $i=1,2,3,4,5$. By $f(0)=0$ and $f(-x)=-f(x)$ for all $x \in X$, we get $A^{0}(x)=A^{0}=0$, and the function $f$ is odd. Thus we have $A^{4}(x)=A^{2}(x)=0$. It follows that $f(x)=A^{5}(x)+A^{3}(x)+A^{1}(x)$. By (2.7) and $A^{n}(r x)=r^{n} A^{n}(x)$ whenever $x \in X$ and $r \in \mathbb{Q}$, we obtain $2^{5} A^{3}(x)+2^{5} A^{1}(x)=2^{3} A^{3}(x)+2 A^{1}(x)$. Hence $A^{1}(x)=-4 A^{3}(x) / 5$, and so $A^{3}(x)=A^{1}(x)=0$ for all $x \in X$. Therefore, $f(x)=A^{5}(x)$.

Conversely, assume that $f(x)=A^{5}(x)$ for all $x \in X$, where $A^{5}(x)$ is the diagonal of the 5-additive symmetric map $A_{5}: X^{5} \rightarrow Y$. From $A^{5}(x+y)=A^{5}(x)+A^{5}(y)+5 A^{4,1}(x, y)+$ $10 A^{3,2}(x, y)+10 A^{2,3}(x, y)+5 A^{1,4}(x, y), A^{5}(r x)=r^{5} A^{5}(x), A^{4,1}(x, r y)=r A^{4,1}(x, y)$, $A^{3,2}(x, r y)=r^{2} A^{3,2}(x, y), A^{2,3}(x, r y)=r^{3} A^{2,3}(x, y)$, and $A^{1,4}(x, r y)=r^{4} A^{1,4}(x, y)(x, y \in$ $X, r \in \mathbb{Q}$ ), we see that $f$ satisfies (1.4), which completes the proof of Theorem 2.1.

Theorem 2.2. A function $f: X \rightarrow Y$ is a solution of the functional equation (1.5) if and only if $f$ is of the form $f(x)=A^{6}(x)$ for all $x \in X$, where $A^{6}(x)$ is the diagonal of the 6-additive symmetric map $A_{6}: X^{6} \rightarrow Y$.

Proof. Assume that $f$ satisfies the functional equation (1.5). Replacing $x=y=0$ in (1.5), one gets $f(0)=0$. Substituting $y$ by $-y$ in (1.5) and subtracting the resulting equation from (1.5) 
and then $y$ by $x$, we obtain $f(-x)=f(x)$. Replacing $(x, y)$ with $(0,2 x)$ and $(3 x, x)$ in (1.5), respectively, we get

$$
\begin{gathered}
f(6 x)-6 f(4 x)-345 f(2 x)=0 \\
f(6 x)-6 f(5 x)+15 f(4 x)-20 f(3 x)+15 f(2 x)-726 f(x)=0
\end{gathered}
$$

for all $x \in X$. Subtracting (2.10) and (2.11), we find

$$
6 f(5 x)-21 f(4 x)+20 f(3 x)-360 f(2 x)+726 f(x)=0
$$

for all $x \in X$. Replacing $(x, y)$ with $(2 x, x)$ in (1.5) and from $f(0)=0$ and $f(-x)=f(x)$ and then multiplying by 6 , we obtain

$$
6 f(5 x)-36 f(4 x)+90 f(3 x)-120 f(2 x)-4224 f(x)=0
$$

for all $x \in X$. Subtracting (2.12) and (2.13), one gets

$$
15 f(4 x)-70 f(3 x)-240 f(2 x)+4950 f(x)=0
$$

for all $x \in X$. Replacing $(x, y)$ with $(0, x)$ (and then multiplying by 10$)$ and $(x, y)$ with $(x, x)$ (and then multiplying by 15 ) in (1.5), respectively, we find

$$
20 f(3 x)-120 f(2 x)-6900 f(x)=0
$$

by $f(0)=0$ and $f(-x)=f(x)$, as well as

$$
15 f(4 x)-90 f(3 x)+240 f(2 x)-11190 f(x)=0
$$

by $f(0)=0$ and $f(-x)=f(x)$. Subtracting (2.14) and (2.16), one gets

$$
20 f(3 x)-480 f(2 x)+16140 f(x)=0
$$

for all $x \in X$. Subtracting (2.15) and (2.17), we have

$$
360 f(2 x)=23040 f(x)
$$

for all $x \in X$. Hence

$$
f(2 x)=2^{6} f(x)
$$

for all $x \in X$. 
On the other hand, one can rewrite the functional equation (1.5) in the form

$$
\begin{aligned}
f(x) & -\frac{1}{20} f(x+3 y)+\frac{3}{10} f(x+2 y)-\frac{3}{4} f(x+y)-\frac{3}{4} f(x-y) \\
+ & \frac{3}{10} f(x-2 y)-\frac{1}{20} f(x-3 y)+36 f(y)=0
\end{aligned}
$$

for all $x \in X$. By [29, Theorems 3.5 and 3.6], $f$ is a generalized polynomial function of degree at most 6 , that is $f$ is of the form,

$$
f(x)=A^{6}(x)+A^{5}(x)+A^{4}(x)+A^{3}(x)+A^{2}(x)+A^{1}(x)+A^{0}(x), \quad \text { for all } x \in X,
$$

where $A^{0}(x)=A^{0}$ is an arbitrary element of $Y$ and $A^{i}(x)$ is the diagonal of the $i$-additive symmetric map $A_{i}: X^{i} \rightarrow Y$ for $i=1,2,3,4,5,6$. By $f(0)=0$ and $f(-x)=f(x)$ for all $x \in X$, we get $A^{0}(x)=A^{0}=0$ and the function $f$ is even. Thus we have $A^{5}(x)=0, A^{3}(x)=0$, and $A^{1}(x)=0$. It follows that $f(x)=A^{6}(x)+A^{4}(x)+A^{2}(x)$. By (2.19) and $A^{n}(r x)=r^{n} A^{n}(x)$ whenever $x \in X$ and $r \in \mathbb{Q}$, we obtain $2^{6} A^{4}(x)+2^{6} A^{2}(x)=2^{4} A^{4}(x)+2^{2} A^{2}(x)$. Hence $A^{2}(x)=$ $-4 A^{4}(x) / 5$, and so $A^{2}(x)=A^{4}(x)=0$ for all $x \in X$. Therefore, $f(x)=A^{6}(x)$.

Conversely, assume that $f(x)=A^{6}(x)$ for all $x \in X$, where $A^{6}(x)$ is the diagonal of the 6-additive symmetric map $A_{6}: X^{6} \rightarrow Y$. From $A^{6}(x+y)=A^{6}(x)+A^{6}(y)+6 A^{5,1}(x, y)+$ $15 A^{4,2}(x, y)+20 A^{3,3}(x, y)+15 A^{2,4}(x, y)+6 A^{1,5}(x, y), A^{6}(r x)=r^{6} A^{6}(x), A^{5,1}(x, r y)=$ $r A^{5,1}(x, y), A^{4,2}(x, r y)=r^{2} A^{4,2}(x, y), A^{3,3}(x, r y)=r^{3} A^{3,3}(x, y), A^{2,4}(x, r y)=r^{4} A^{2,4}(x, y)$, and $A^{1,5}(x, r y)=r^{5} A^{1,5}(x, y)(x, y \in X, r \in \mathbb{Q})$, we see that $f$ satisfies (1.5), which completes the proof of Theorem 2.2.

\section{Stability of the Quintic Functional Equation}

Throughout this section, unless otherwise explicitly stated, we will assume that $X$ is a linear space, $Y$ is a $(\beta, p)$-Banach space with $(\beta, p)$-norm $\|\cdot\|_{Y}$. Let $K$ be the modulus of concavity of $\|\cdot\|_{Y}$. We will establish the following stability for the quintic functional equation in quasi$\beta$-normed spaces. For notational convenience, given a function $f: X \rightarrow Y$, we define the difference operator

$$
\begin{aligned}
D_{q} f(x, y):= & f(x+3 y)-5 f(x+2 y)+10 f(x+y)-10 f(x) \\
& +5 f(x-y)-f(x-2 y)-120 f(y)
\end{aligned}
$$

for all $x, y \in X$.

Lemma 3.1. Let $j \in\{-1,1\}$ be fixed, $s, a \in \mathbb{N}$ with $a \geq 2$ and $\psi: X \rightarrow[0, \infty)$ a function such that there exists an $L<1$ with $\psi\left(a^{j} x\right) \leq a^{j s \beta} L \psi(x)$ for all $x \in X$. Let $f: X \rightarrow Y$ be a mapping satisfying

$$
\left\|f(a x)-a^{s} f(x)\right\|_{Y} \leq \psi(x)
$$


for all $x \in X$, then there exists a uniquely determined mapping $F: X \rightarrow Y$ such that $F(a x)=a^{s} F(x)$ and

$$
\|f(x)-F(x)\|_{Y} \leq \frac{1}{a^{s \beta}\left|1-L^{j}\right|} \psi(x)
$$

for all $x \in X$.

Proof. Consider the set

$$
\Omega:=\{g \mid g: X \longrightarrow Y\},
$$

and introduce the generalized metric on $\Omega$,

$$
d(g, h)=\inf \left\{\mu>0 \mid\|g(x)-h(x)\|_{Y} \leq \mu \psi(x) \quad \forall x \in X\right\} .
$$

It is easy to show that $(\Omega, d)$ is a complete generalized metric space (see [8-10]).

Define a function $J: \Omega \rightarrow \Omega$ by $J g(x)=a^{-j s} g\left(a^{j} x\right)$ for all $x \in X$. Let $g, h \in \Omega$ be given such that $d(g, h)<\varepsilon$, by the definition,

$$
\|g(x)-h(x)\|_{Y} \leq \varepsilon \psi(x), \quad \forall x \in X
$$

Hence

$$
\|J g(x)-J h(x)\|_{Y}=a^{-j s \beta}\left\|g\left(a^{j} x\right)-h\left(a^{j} x\right)\right\|_{Y} \leq a^{-j s \beta} \varepsilon \psi\left(a^{j} x\right) \leq L \varepsilon \psi(x)
$$

for all $x \in X$. By definition, $d(J g, J h) \leq L \varepsilon$. Therefore,

$$
d(J g, J h) \leq L d(g, h), \quad \forall g, h \in \Omega
$$

This means that $J$ is a strictly contractive self-mapping of $\Omega$ with Lipschitz constant $L$.

It follows from (3.2) that

$$
d(f, J f) \leq \begin{cases}\frac{1}{a^{s \beta}} \psi(x), & \text { if } j=1, \\ \frac{L}{a^{s \beta}} \psi(x), & \text { if } j=-1,\end{cases}
$$

for all $x \in X$. Therefore, by [10, Theorem 1.3], $J$ has a unique fixed point $F: X \rightarrow Y$ in the set $\Delta=\{g \in \Omega: d(g, f)<\infty\}$. This implies that $F(a x)=a^{s} F(x)$ and

$$
F(x):=\lim _{n \rightarrow \infty} J^{n} f(x)=\lim _{n \rightarrow \infty} a^{-j n s} f\left(a^{j n} x\right)
$$


for all $x \in X$. Moreover,

$$
d(f, F) \leq \frac{1}{1-L} d(f, J f) \leq \frac{1}{a^{s \beta}\left|1-L^{j}\right|} .
$$

This implies that the inequality (3.3) holds.

To prove the uniqueness of the mapping $F$, assume that there exists another mapping $G: X \rightarrow Y$ which satisfies (3.3) and $G(a x)=a^{s} G(x)$ for all $x \in X$. Fix $x \in X$. Clearly, $F\left(a^{j n} x\right)=a^{j s n} F(x)$ and $G\left(a^{j n} x\right)=a^{j s n} G(x)$ for all $n \in \mathbb{N}$. Thus

$$
\|F(x)-G(x)\|_{Y} \leq K\left\|\frac{F\left(a^{j n} x\right)}{a^{j s n}}-\frac{f\left(a^{j n} x\right)}{a^{j s n}}\right\|_{Y}+K\left\|\frac{G\left(a^{j n} x\right)}{a^{j s n}}-\frac{f\left(a^{j n} x\right)}{a^{j s n}}\right\|_{Y} \leq \frac{2 K L^{n}}{a^{s \beta}\left|1-L^{j}\right|} \psi(x) .
$$

Since, for every $x \in X, \lim _{n \rightarrow \infty}\left(\left(2 K L^{n}\right) /\left(a^{s \beta}\left|1-L^{j}\right|\right)\right) \psi(x)=0$, we get $G=F$. This completes the proof.

Theorem 3.2. Let $j \in\{-1,1\}$ be fixed, and let $\varphi: X \times X \rightarrow[0, \infty)$ be a function such that there exists an $L<1$ with $\varphi\left(2^{j} x, 2^{j} y\right) \leq 32^{j \beta} L \varphi(x, y)$ for all $x, y \in X$. Let $f: X \rightarrow Y$ be a mapping satisfying

$$
\left\|D_{q} f(x, y)\right\|_{Y} \leq \varphi(x, y)
$$

for all $x, y \in X$. Then there exists a unique quintic mapping $Q: X \rightarrow Y$ such that

$$
\|f(x)-Q(x)\|_{Y} \leq \frac{1}{32^{\beta} \mid 1-L^{j \mid}} \tilde{\varphi}(x)
$$

for all $x \in X$, where

$$
\begin{aligned}
\tilde{\varphi}(x):=\frac{K^{2}}{120^{\beta}}[ & K^{3} \varphi(3 x, x)+15^{\beta} \varphi(0, x)+K^{3} \varphi(0,2 x)+\left(\frac{K^{4}}{24^{\beta}}+\frac{K^{3}}{8^{\beta}}\right)(\varphi(0,2 x)+\varphi(2 x,-2 x)) \\
& +5^{\beta} K^{2} \varphi(2 x, x)+11^{\beta} K \varphi(x, x)+\left(\frac{K^{3}}{12^{\beta}}+\frac{K^{2}}{24^{\beta}}+\frac{11^{\beta} K^{2}}{24^{\beta}}+\frac{5^{\beta} K}{4^{\beta}}\right) \varphi(0,0) \\
& \left.+\left(\frac{11^{\beta} K^{3}}{12^{\beta}}+\frac{5^{\beta} K^{3}}{8^{\beta}}\right)(\varphi(0, x)+\varphi(x,-x))\right]
\end{aligned}
$$

for all $x \in X$.

Proof. Replacing $x=y=0$ in (3.13), we get

$$
\|f(0)\|_{Y} \leq \frac{1}{120^{\beta}} \varphi(0,0) .
$$


Journal of Inequalities and Applications

Replacing $x$ and $y$ by 0 and $x$ in (3.13), respectively, we get

$$
\|f(3 x)-5 f(2 x)+10 f(x)-10 f(0)+5 f(-x)-f(-2 x)-120 f(x)\|_{Y} \leq \varphi(0, x)
$$

for all $x \in X$. Replacing $x$ and $y$ by $x$ and $-x$ in (3.13), respectively, we have

$$
\|f(-2 x)-5 f(-x)+10 f(0)-10 f(x)+5 f(2 x)-f(3 x)-120 f(-x)\|_{Y} \leq \varphi(x,-x)
$$

for all $x \in X$. By (3.17) and (3.18), we obtain

$$
\|f(x)+f(-x)\|_{Y} \leq \frac{K}{120^{\beta}}(\varphi(0, x)+\varphi(x,-x))
$$

for all $x \in X$. Replacing $x$ and $y$ by $3 x$ and $x$ in (3.13), respectively, we get

$$
\|f(6 x)-5 f(5 x)+10 f(4 x)-10 f(3 x)+5 f(2 x)-121 f(x)\|_{Y} \leq \varphi(3 x, x)
$$

for all $x \in X$. Replacing $x$ and $y$ by 0 and $2 x$ in (3.13), respectively, we find

$$
\|f(6 x)-5 f(4 x)-10 f(0)+5 f(-2 x)-f(-4 x)-110 f(2 x)\|_{Y} \leq \varphi(0,2 x)
$$

for all $x \in X$. By (3.20) and (3.21), we obtain

$$
\begin{aligned}
& \|5 f(5 x)-14 f(4 x)+10 f(3 x)+121 f(x)-120 f(2 x)+5 f(2 x)+5 f(-2 x)-10 f(0)\|_{Y} \\
& \quad \leq K \varphi(3 x, x)+K \varphi(0,2 x)
\end{aligned}
$$

for all $x \in X$. By (3.16), (3.19), and (3.22), we have

$$
\begin{aligned}
& \|5 f(5 x)-14 f(4 x)+10 f(3 x)+121 f(x)-120 f(2 x)\|_{Y} \\
& \quad \leq K^{2} \varphi(3 x, x)+K^{2} \varphi(0,2 x)+\frac{K^{3}}{24^{\beta}}(\varphi(0,2 x)+\varphi(2 x,-2 x))+\frac{K^{2}}{12^{\beta}} \varphi(0,0)
\end{aligned}
$$

for all $x \in X$. Replacing $x$ and $y$ by $2 x$ and $x$ in (3.13), respectively, we get

$$
\|f(5 x)-5 f(4 x)+10 f(3 x)-10 f(2 x)-115 f(x)-f(0)\|_{Y} \leq \varphi(2 x, x)
$$

for all $x \in X$. Using (3.16), we have

$$
\|f(5 x)-5 f(4 x)+10 f(3 x)-10 f(2 x)-115 f(x)\|_{Y} \leq K \varphi(2 x, x)+\frac{K}{120^{\beta}} \varphi(0,0)
$$


for all $x \in X$. Hence

$$
\|5 f(5 x)-25 f(4 x)+50 f(3 x)-50 f(2 x)-575 f(x)\|_{Y} \leq 5^{\beta} K \varphi(2 x, x)+\frac{K}{24^{\beta}} \varphi(0,0)
$$

for all $x \in X$. By (3.23) and (3.26), we get

$$
\begin{aligned}
\|11 f(4 x)-40 f(3 x)-70 f(2 x)+696 f(x)\|_{Y} \\
\leq K^{3} \varphi(3 x, x)+K^{3} \varphi(0,2 x)+\frac{K^{4}}{24^{\beta}}(\varphi(0,2 x)+\varphi(2 x,-2 x)) \\
\quad+\left(\frac{K^{3}}{12^{\beta}}+\frac{K^{2}}{24^{\beta}}\right) \varphi(0,0)+5^{\beta} K^{2} \varphi(2 x, x)
\end{aligned}
$$

for all $x \in X$. Replacing $x$ and $y$ by $x$ and $x$ in (3.13), respectively, we have

$$
\|f(4 x)-5 f(3 x)+10 f(2 x)+5 f(0)-f(-x)-130 f(x)\|_{Y} \leq \varphi(x, x)
$$

for all $x \in X$. By (3.16), (3.19), and (3.28), we have

$$
\|f(4 x)-5 f(3 x)+10 f(2 x)-129 f(x)\|_{Y} \leq K \varphi(x, x)+\frac{K^{3}}{120^{\beta}}(\varphi(0, x)+\varphi(x,-x))+\frac{K^{2}}{24^{\beta}} \varphi(0,0)
$$

for all $x \in X$. Thus

$$
\begin{aligned}
& \|11 f(4 x)-55 f(3 x)+110 f(2 x)-1419 f(x)\|_{Y} \\
& \quad \leq 11^{\beta} K \varphi(x, x)+\frac{11^{\beta} K^{3}}{120^{\beta}}(\varphi(0, x)+\varphi(x,-x))+\frac{11^{\beta} K^{2}}{24^{\beta}} \varphi(0,0)
\end{aligned}
$$

for all $x \in X$. By (3.27) and (3.30), we obtain

$$
\begin{aligned}
&\|15 f(3 x)-180 f(2 x)+2115 f(x)\|_{Y} \\
& \leq K^{4} \varphi(3 x, x)+K^{4} \varphi(0,2 x)+\frac{K^{5}}{24^{\beta}}(\varphi(0,2 x)+\varphi(2 x,-2 x)) \\
&+\left(\frac{K^{4}}{12^{\beta}}+\frac{K^{3}}{24^{\beta}}+\frac{11^{\beta} K^{3}}{24^{\beta}}\right) \varphi(0,0)+5^{\beta} K^{3} \varphi(2 x, x)+11^{\beta} K^{2} \varphi(x, x) \\
&+\frac{11^{\beta} K^{4}}{120^{\beta}}(\varphi(0, x)+\varphi(x,-x))
\end{aligned}
$$


for all $x \in X$. By (3.16), (3.17), and (3.19), we have

$$
\begin{aligned}
\| f(3 x) & -4 f(2 x)-115 f(x) \|_{Y} \\
\leq & K \varphi(0, x)+\frac{K^{2}}{12^{\beta}} \varphi(0,0)+\frac{K^{4}}{24^{\beta}}(\varphi(0, x)+\varphi(x,-x))+\frac{K^{4}}{120^{\beta}}(\varphi(0,2 x)+\varphi(2 x,-2 x))
\end{aligned}
$$

for all $x \in X$. Hence

$$
\begin{aligned}
& \|15 f(3 x)-60 f(2 x)-1725 f(x)\|_{Y} \\
& \quad \leq 15^{\beta} K \varphi(0, x)+\frac{5^{\beta} K^{2}}{4^{\beta}} \varphi(0,0)+\frac{5^{\beta} K^{4}}{8^{\beta}}(\varphi(0, x)+\varphi(x,-x))+\frac{K^{4}}{8^{\beta}}(\varphi(0,2 x)+\varphi(2 x,-2 x))
\end{aligned}
$$

for all $x \in X$. By (3.31) and (3.33), we get

$$
\begin{aligned}
& \left\|f(2 x)-2^{5} f(x)\right\|_{Y} \\
& \leq \frac{K^{2}}{120^{\beta}}\left[K^{3} \varphi(3 x, x)+15^{\beta} \varphi(0, x)+K^{3} \varphi(0,2 x)+\left(\frac{K^{4}}{24^{\beta}}+\frac{K^{3}}{8^{\beta}}\right)(\varphi(0,2 x)+\varphi(2 x,-2 x))\right. \\
& +5^{\beta} K^{2} \varphi(2 x, x)+11^{\beta} K \varphi(x, x)+\left(\frac{K^{3}}{12^{\beta}}+\frac{K^{2}}{24^{\beta}}+\frac{11^{\beta} K^{2}}{24^{\beta}}+\frac{5^{\beta} K}{4^{\beta}}\right) \varphi(0,0) \\
& \left.+\left(\frac{11^{\beta} K^{3}}{120^{\beta}}+\frac{5^{\beta} K^{3}}{8^{\beta}}\right)(\varphi(0, x)+\varphi(x,-x))\right] \\
& =\tilde{\varphi}(x)
\end{aligned}
$$

for all $x \in X$. By Lemma 3.1, there exists a unique mapping $Q: X \rightarrow Y$ such that $Q(2 x)=$ $2^{5} Q(x)$ and

$$
\|f(x)-Q(x)\|_{Y} \leq \frac{1}{32^{\beta}\left|1-L^{j}\right|} \tilde{\varphi}(x)
$$

for all $x \in X$. It remains to show that $Q$ is a quintic map. By (3.13), we have

$$
\left\|\frac{D_{q} f\left(2^{j n} x, 2^{j n} y\right)}{32^{j n}}\right\|_{Y} \leq 32^{-j n \beta} \varphi\left(2^{j n} x, 2^{j n} y\right) \leq 32^{-j n \beta}\left(32^{j \beta} L\right)^{n} \varphi(x, y)=L^{n} \varphi(x, y)
$$

for all $x, y \in X$ and $n \in \mathbb{N}$. So

$$
\left\|D_{q} Q(x, y)\right\|_{Y}=0
$$

for all $x, y \in X$. Thus the mapping $Q: X \rightarrow Y$ is quintic, as desired. 
Corollary 3.3. Let $X$ be a quasi- $\alpha$-normed space with quasi- $\alpha$-norm $\|\cdot\|_{X}$, and let $Y$ be a $(\beta, p)$ Banach space with $(\beta, p)$-norm $\|\cdot\|_{Y}$. Let $\delta, r, s$ be positive numbers with $\lambda:=r+s \neq 5 \beta / \alpha$ and $f: X \rightarrow Y$ a mapping satisfying

$$
\left\|D_{q} f(x, y)\right\|_{Y} \leq \delta\|x\|_{X}^{r}\|y\|_{X}^{s}
$$

for all $x, y \in X$. Then there exists a unique quintic mapping $Q: X \rightarrow Y$ such that

$$
\|f(x)-Q(x)\|_{Y} \leq \begin{cases}\frac{\delta \varepsilon_{r, s}}{32^{\beta}-2^{\alpha \lambda}}\|x\|_{X}^{\lambda}, & \lambda \in\left(0, \frac{5 \beta}{\alpha}\right), \\ \frac{2^{\lambda \alpha} \delta \varepsilon_{r, s}}{32^{\beta}\left(2^{\lambda \alpha}-32^{\beta}\right)}\|x\|_{X}^{\lambda}, & \lambda \in\left(\frac{5 \beta}{\alpha}, \infty\right),\end{cases}
$$

for all $x \in X$, where

$$
\varepsilon_{r, s}:=\frac{K^{2}}{120^{\beta}}\left(K^{3} 3^{\alpha r}+\frac{K^{4} 2^{\alpha \lambda}}{24^{\beta}}+\frac{K^{3} 2^{\alpha \lambda}}{8^{\beta}}+K^{2} 5^{\beta} 2^{\alpha r}+11^{\beta}+\frac{K^{3} 11^{\beta}}{120^{\beta}}+\frac{K^{3} 5^{\beta}}{8^{\beta}}\right) .
$$

Corollary 3.4. Let $X$ be a quasi- $\alpha$-normed space with quasi- $\alpha$-norm $\|\cdot\|_{X}$, and let $Y$ be a $(\beta, p)$ Banach space with $(\beta, p)$-norm $\|\cdot\|_{Y}$. Let $\delta, \lambda$ be positive numbers with $\lambda \neq 5 \beta / \alpha$ and $f: X \rightarrow Y a$ mapping satisfying

$$
\left\|D_{q} f(x, y)\right\|_{Y} \leq \delta\left(\|x\|_{X}^{\lambda}+\|y\|_{X}^{\lambda}\right)
$$

for all $x, y \in X$. Then there exists a unique quintic mapping $Q: X \rightarrow Y$ such that

$$
\|f(x)-Q(x)\|_{Y} \leq \begin{cases}\frac{\delta \varepsilon_{\lambda}}{32^{\beta}-2^{\alpha \lambda}}\|x\|_{X}^{\lambda}, & \lambda \in\left(0, \frac{5 \beta}{\alpha}\right), \\ \frac{2^{\lambda \alpha} \delta \varepsilon_{\lambda}}{32^{\beta}\left(2^{\lambda \alpha}-32^{\beta}\right)}\|x\|_{X}^{\lambda}, & \lambda \in\left(\frac{5 \beta}{\alpha}, \infty\right),\end{cases}
$$

for all $x \in X$, where

$$
\begin{gathered}
\mathcal{E}_{\lambda}:=\frac{K^{2}}{120^{\beta}}\left[K^{3}\left(3^{\alpha \lambda}+2^{\alpha \lambda}+1\right)+K^{2} 5^{\beta}\left(2^{\alpha \lambda}+1\right)+2 K 11^{\beta}+15^{\beta}\right. \\
\left.+3\left(\frac{K^{4}}{24^{\beta}}+\frac{K^{3}}{8^{\beta}}\right) 2^{\alpha \lambda}+3\left(\frac{K^{3} 11^{\beta}}{120^{\beta}}+\frac{K^{3} 5^{\beta}}{8^{\beta}}\right)\right] .
\end{gathered}
$$

Corollary 3.5. Let $X$ be a quasi- $\alpha$-normed space with quasi- $\alpha$-norm $\|\cdot\|_{X}$, and let $Y$ be a $(\beta, p)$ Banach space with $(\beta, p)$-norm $\|\cdot\|_{\gamma}$. Let $\delta, r, s$ be positive numbers with $\lambda:=r+s \neq 5 \beta / \alpha$ and $f: X \rightarrow Y$ a mapping satisfying

$$
\left\|D_{q} f(x, y)\right\|_{Y} \leq \delta\left[\|x\|_{X}^{r}\|y\|_{X}^{s}+\left(\|x\|_{X}^{r+s}+\|y\|_{X}^{r+s}\right)\right]
$$


for all $x, y \in X$. Then there exists a unique quintic mapping $Q: X \rightarrow Y$ such that

$$
\|f(x)-Q(x)\|_{Y} \leq \begin{cases}\frac{\delta\left(\varepsilon_{r, s}+\varepsilon_{\curlywedge}\right)}{32^{\beta}-2^{\alpha \lambda}}\|x\|_{X}^{\lambda}, & \lambda \in\left(0, \frac{5 \beta}{\alpha}\right), \\ \frac{2^{\lambda \alpha} \delta\left(\varepsilon_{r, s}+\varepsilon_{\curlywedge}\right)}{32^{\beta}\left(2^{\lambda \alpha}-32^{\beta}\right)}\|x\|_{X}^{\lambda}, & \lambda \in\left(\frac{5 \beta}{\alpha}, \infty\right),\end{cases}
$$

for all $x \in X$, where $\varepsilon_{r, s}$ and $\varepsilon_{\lambda}$ are defined as in Corollaries 3.3 and 3.4.

The following example shows that the assumption $\lambda \neq 5 \beta / \alpha$ cannot be omitted in Corollary 3.4. This example is a modification of the example of Gajda [6] for the additive functional inequality (see also [7]).

Example 3.6. Let $\phi: \mathbb{R} \rightarrow \mathbb{R}$ be defined by

$$
\phi(x)= \begin{cases}x^{5}, & \text { for }|x|<1 \\ 1, & \text { for }|x| \geq 1\end{cases}
$$

Consider that the function $f: \mathbb{R} \rightarrow \mathbb{R}$ is defined by

$$
f(x)=\sum_{n=0}^{\infty} 4^{-5 n} \phi\left(4^{n} x\right)
$$

for all $x \in \mathbb{R}$. Then $f$ satisfies the functional inequality

$$
\begin{aligned}
& |f(x+3 y)-5 f(x+2 y)+10 f(x+y)-10 f(x)+5 f(x-y)-f(x-2 y)-120 f(y)| \\
& \quad \leq \frac{152 \cdot 1024^{3}}{1023}\left(|x|^{5}+|y|^{5}\right)
\end{aligned}
$$

for all $x, y \in \mathbb{R}$, but there do not exist a quintic mapping $Q: \mathbb{R} \rightarrow \mathbb{R}$ and a constant $d>0$ such that $|f(x)-Q(x)| \leq d|x|^{5}$ for all $x \in \mathbb{R}$.

Proof. It is clear that $f$ is bounded by $1024 / 1023$ on $\mathbb{R}$. If $|x|^{5}+|y|^{5}=0$ or $|x|^{5}+|y|^{5} \geq 1 / 1024$, then

$$
\left|D_{q} f(x, y)\right| \leq \frac{152 \cdot 1024}{1023} \leq \frac{152 \cdot 1024^{2}}{1023}\left(|x|^{5}+|y|^{5}\right)
$$

Now suppose that $0<|x|^{5}+|y|^{5}<1 / 1024$. Then there exists a nonnegative integer $k$ such that

$$
\frac{1}{1024^{k+2}} \leq|x|^{5}+|y|^{5}<\frac{1}{1024^{k+1}}
$$


Hence $1024^{k}|x|^{5}<1 / 1024,1024^{k}|y|^{5}<1 / 1024$, and $4^{n}(x+3 y), 4^{n}(x+2 y), 4^{n}(x-2 y), 4^{n}(x+$ $y), 4^{n}(x-y), 4^{n} x, 4^{n} y \in(-1,1)$ for all $n=0,1, \ldots, k-1$. Hence, for $n=0,1, \ldots, k-1$,

$$
\begin{aligned}
\phi\left(4^{n}(x+3 y)\right)-5 \phi\left(4^{n}(x+2 y)\right) & +10 \phi\left(4^{n}(x+y)\right)-10 \phi\left(4^{n} x\right)+5 \phi\left(4^{n}(x-y)\right) \\
-\phi\left(4^{n}(x-2 y)\right)-120 \phi\left(4^{n} y\right) & =0 .
\end{aligned}
$$

From the definition of $f$ and the inequality (3.50), we obtain that

$$
\begin{aligned}
\left|D_{q} f(x, y)\right|= & \mid \sum_{n=0}^{\infty} 4^{-5 n} \phi\left(4^{n}(x+3 y)\right)-5 \sum_{n=0}^{\infty} 4^{-5 n} \phi\left(4^{n}(x+2 y)\right)+10 \sum_{n=0}^{\infty} 4^{-5 n} \phi\left(4^{n}(x+y)\right) \\
& -10 \sum_{n=0}^{\infty} 4^{-5 n} \phi\left(4^{n} x\right)+5 \sum_{n=0}^{\infty} 4^{-5 n} \phi\left(4^{n}(x-y)\right)-\sum_{n=0}^{\infty} 4^{-5 n} \phi\left(4^{n}(x-2 y)\right) \\
& -120 \sum_{n=0}^{\infty} 4^{-5 n} \phi\left(4^{n} y\right) \mid \\
\leq & \sum_{n=0}^{\infty} 4^{-5 n} \mid \phi\left(4^{n}(x+3 y)\right)-5 \phi\left(4^{n}(x+2 y)\right)+10 \phi\left(4^{n}(x+y)\right)-10 \phi\left(4^{n} x\right) \\
& +5 \phi\left(4^{n}(x-y)\right)-\phi\left(4^{n}(x-2 y)\right)-120 \phi\left(4^{n} y\right) \mid \\
\leq & \sum_{n=k}^{\infty} 4^{-5 n} \cdot 152=\frac{152 \cdot 4^{5(1-k)}}{1023} \leq \frac{152 \cdot 1024^{3}}{1023}\left(|x|^{5}+|y|^{5}\right) .
\end{aligned}
$$

Therefore, $f$ satisfies (3.48) for all $x, y \in \mathbb{R}$. Now, we claim that the functional equation (1.4) is not stable for $\lambda=5$ in Corollary $3.4(\alpha=\beta=p=1)$. Suppose on the contrary that there exists a quintic mapping $Q: \mathbb{R} \rightarrow \mathbb{R}$ and constant $d>0$ such that $|f(x)-Q(x)| \leq d|x|^{5}$ for all $x \in \mathbb{R}$. Then there exists a constant $c \in \mathbb{R}$ such that $Q(x)=c x^{5}$ for all rational numbers $x$ (see [7]). So we obtain that

$$
|f(x)| \leq(d+|c|)|x|^{5}
$$

for all $x \in \mathbb{Q}$. Let $m \in \mathbb{N}$ with $m+1>d+|c|$. If $x$ is a rational number in $\left(0,4^{-m}\right)$, then $4^{n} x \in(0,1)$ for all $n=0,1, \ldots, m$, and for this $x$ we get

$$
f(x)=\sum_{n=0}^{\infty} \frac{\phi\left(4^{n} x\right)}{4^{5 n}} \geq \sum_{n=0}^{m} \frac{\left(4^{n} x\right)^{5}}{4^{5 n}}=(m+1) x^{5}>(d+|c|) x^{5},
$$

which contradicts (3.53). 


\section{Stability of the Sextic Functional Equation}

Throughout this section, unless otherwise explicitly stated, we will assume that $X$ is a linear space, $Y$ is a $(\beta, p)$-Banach space with $(\beta, p)$-norm $\|\cdot\|_{\gamma}$. Let $K$ be the modulus of concavity of $\|\cdot\|_{\gamma}$. We will establish the following stability for the sextic functional equation in quasi$\beta$-normed spaces. For notational convenience, given a function $f: X \rightarrow Y$, we define the difference operator

$$
\begin{aligned}
D_{s} f(x, y):= & f(x+3 y)-6 f(x+2 y)+15 f(x+y)-20 f(x)+15 f(x-y)-6 f(x-2 y) \\
& +f(x-3 y)-720 f(y)
\end{aligned}
$$

for all $x, y \in X$.

Theorem 4.1. Let $j \in\{-1,1\}$ be fixed, and let $\varphi: X \times X \rightarrow[0, \infty)$ be a function such that there exists an $L<1$ with $\varphi\left(2^{j} x, 2^{j} y\right) \leq 64^{j \beta} L \varphi(x, y)$ for all $x, y \in X$. Let $f: X \rightarrow Y$ be a mapping satisfying

$$
\left\|D_{s} f(x, y)\right\|_{Y} \leq \varphi(x, y)
$$

for all $x, y \in X$. Then there exists a unique sextic mapping $S: X \rightarrow Y$ such that

$$
\|f(x)-S(x)\|_{Y} \leq \frac{1}{64^{\beta}\left|1-L^{j}\right|} \tilde{\varphi}(x)
$$

for all $x \in X$, where

$$
\begin{aligned}
\tilde{\varphi}(x):=\frac{1}{360^{\beta}}[ & \frac{10^{\beta} K^{6}+K^{4}\left(225^{\beta}+36^{\beta}+1\right)+K^{3} 200^{\beta}}{720^{\beta}} \varphi(0,0)+\frac{K^{7}}{2^{\beta}} \varphi(0,2 x)+K^{5} \varphi(3 x, x) \\
& +K^{5} 6^{\beta} \varphi(2 x, x)+K^{3} 15^{\beta} \varphi(x, x)+\frac{K^{9}}{1440^{\beta}}(\varphi(6 x, 6 x)+\varphi(6 x,-6 x)) \\
& +\frac{K^{10}}{240^{\beta}}(\varphi(4 x, 4 x)+\varphi(4 x,-4 x))+\frac{K^{6}}{12^{\beta}}(\varphi(3 x, 3 x)+\varphi(3 x,-3 x)) \\
& +\frac{K^{10}+K^{6}\left(2^{\beta}+8^{\beta}\right)}{96^{\beta}}(\varphi(2 x, 2 x)+\varphi(2 x,-2 x)) \\
& \left.+\frac{K^{6}\left(15^{\beta}+1\right)+K^{5} 25^{\beta}}{120^{\beta}}(\varphi(x, x)+\varphi(x,-x))+K^{2} 10^{\beta} \varphi(0, x)\right]
\end{aligned}
$$

for all $x \in X$. 
Proof. Replacing $x=y=0$ in (4.2), we get

$$
\|f(0)\|_{Y} \leq \frac{1}{720^{\beta}} \varphi(0,0) .
$$

Replacing $y$ by $-y$ in (4.2), we have

$$
\begin{aligned}
& \| f(x-3 y)-6 f(x-2 y)+15 f(x-y)-20 f(x)+15 f(x+y)-6 f(x+2 y) \\
& \quad+f(x+3 y)-720 f(-y) \|_{Y} \\
& \leq \varphi(x,-y)
\end{aligned}
$$

for all $x, y \in X$. By (4.2) and (4.6), we get

$$
\|f(x)-f(-x)\|_{Y} \leq \frac{K}{720^{\beta}}(\varphi(x, x)+\varphi(x,-x))
$$

for all $x \in X$. Replacing $x$ and $y$ by 0 and $2 x$ in (4.2), respectively, we get

$$
\|f(6 x)-6 f(4 x)+15 f(2 x)-20 f(0)+15 f(-2 x)-6 f(-4 x)+f(-6 x)-720 f(2 x)\|_{Y} \leq \varphi(0,2 x)
$$

for all $x \in X$. By (4.5), (4.7), and (4.8), we have

$$
\begin{aligned}
\|f(6 x)-6 f(4 x)-345 f(2 x)\|_{Y} \\
\leq \frac{K}{72^{\beta}} \varphi(0,0)+\frac{K^{2}}{2^{\beta}} \varphi(0,2 x)+\frac{K^{4}}{1440^{\beta}}(\varphi(6 x, 6 x)+\varphi(6 x,-6 x)) \\
\quad+\frac{K^{5}}{240^{\beta}}(\varphi(4 x, 4 x)+\varphi(4 x,-4 x))+\frac{K^{5}}{96^{\beta}}(\varphi(2 x, 2 x)+\varphi(2 x,-2 x))
\end{aligned}
$$

for all $x \in X$. Replacing $x$ and $y$ by $3 x$ and $x$ in (4.2), respectively, we have

$$
\|f(6 x)-6 f(5 x)+15 f(4 x)-20 f(3 x)+15 f(2 x)+f(0)-726 f(x)\|_{Y} \leq \varphi(3 x, x)
$$

for all $x \in X$. Subtracting (4.9)-(4.10) and using (4.5), we obtain

$$
\begin{aligned}
&\|6 f(5 x)-21 f(4 x)+20 f(3 x)-360 f(2 x)+726 f(x)\|_{Y} \\
& \leq \frac{10^{\beta} K^{3}+K}{720^{\beta}} \varphi(0,0)+\frac{K^{4}}{2^{\beta}} \varphi(0,2 x)+\frac{K^{6}}{1440^{\beta}}(\varphi(6 x, 6 x)+\varphi(6 x,-6 x)) \\
&+\frac{K^{7}}{240^{\beta}}(\varphi(4 x, 4 x)+\varphi(4 x,-4 x))+\frac{K^{7}}{96^{\beta}}(\varphi(2 x, 2 x)+\varphi(2 x,-2 x))+K^{2} \varphi(3 x, x)
\end{aligned}
$$


Journal of Inequalities and Applications

for all $x \in X$. Replacing $x$ and $y$ by $2 x$ and $x$ in (4.2), respectively, we have

$$
\|f(5 x)-6 f(4 x)+15 f(3 x)-20 f(2 x)-6 f(0)+f(-x)-705 f(x)\|_{Y} \leq \varphi(2 x, x)
$$

for all $x \in X$. Using (4.5) and (4.7), we get

$$
\begin{aligned}
& \|f(5 x)-6 f(4 x)+15 f(3 x)-20 f(2 x)-704 f(x)\|_{Y} \\
& \leq \frac{K}{120^{\beta}} \varphi(0,0)+\frac{K^{3}}{720^{\beta}}(\varphi(x, x)+\varphi(x,-x))+K^{2} \varphi(2 x, x)
\end{aligned}
$$

for all $x \in X$. Hence

$$
\begin{aligned}
& \|6 f(5 x)-36 f(4 x)+90 f(3 x)-120 f(2 x)-4224 f(x)\|_{Y} \\
& \quad \leq \frac{K}{20^{\beta}} \varphi(0,0)+\frac{K^{3}}{120^{\beta}}(\varphi(x, x)+\varphi(x,-x))+K^{2} 6^{\beta} \varphi(2 x, x)
\end{aligned}
$$

for all $x \in X$. Subtracting (4.11)-(4.14), we have

$$
\begin{aligned}
\|15 f(4 x)-70 f(3 x)-240 f(2 x)+4950 f(x)\|_{Y} \\
\leq \leq \frac{10^{\beta} K^{4}+K^{2}\left(36^{\beta}+1\right)}{720^{\beta}} \varphi(0,0)+\frac{K^{5}}{2^{\beta}} \varphi(0,2 x)+\frac{K^{7}}{1440^{\beta}}(\varphi(6 x, 6 x)+\varphi(6 x,-6 x)) \\
\quad+\frac{K^{8}}{240^{\beta}}(\varphi(4 x, 4 x)+\varphi(4 x,-4 x))+\frac{K^{8}}{96^{\beta}}(\varphi(2 x, 2 x)+\varphi(2 x,-2 x)) \\
\quad+K^{3} \varphi(3 x, x)+\frac{K^{4}}{120^{\beta}}(\varphi(x, x)+\varphi(x,-x))+K^{3} 6^{\beta} \varphi(2 x, x)
\end{aligned}
$$

for all $x \in X$. Replacing $x$ and $y$ by 0 and $x$ in (4.2), respectively, we get

$$
\|f(3 x)-6 f(2 x)-705 f(x)-20 f(0)+15 f(-x)-6 f(-2 x)+f(-3 x)\|_{Y} \leq \varphi(0, x)
$$

for all $x \in X$. By (4.5), (4.7), and (4.16), we have

$$
\begin{aligned}
\| 2 f(3 x) & -12 f(2 x)-690 f(x) \|_{Y} \\
\leq & K \varphi(0, x)+\frac{K^{2}}{36^{\beta}} \varphi(0,0)+\frac{K^{4}}{48^{\beta}}(\varphi(x, x)+\varphi(x,-x))+\frac{K^{5}}{120^{\beta}}(\varphi(2 x, 2 x)+\varphi(2 x,-2 x)) \\
& +\frac{K^{5}}{120^{\beta}}(\varphi(3 x, 3 x)+\varphi(3 x,-3 x))
\end{aligned}
$$


for all $x \in X$. Thus

$$
\begin{aligned}
&\|20 f(3 x)-120 f(2 x)-6900 f(x)\|_{Y} \\
& \leq K 10^{\beta} \varphi(0, x)+\frac{K^{2} 5^{\beta}}{18^{\beta}} \varphi(0,0)+\frac{K^{4} 5^{\beta}}{24^{\beta}}(\varphi(x, x)+\varphi(x,-x)) \\
&+\frac{K^{5}}{12^{\beta}}(\varphi(2 x, 2 x)+\varphi(2 x,-2 x))+\frac{K^{5}}{12^{\beta}}(\varphi(3 x, 3 x)+\varphi(3 x,-3 x))
\end{aligned}
$$

for all $x \in X$. Replacing $x$ and $y$ by $x$ and $x$ in (4.2), respectively, and then using (4.5) and (4.7), we have

$$
\begin{aligned}
& \|f(4 x)-6 f(3 x)+16 f(2 x)-746 f(x)\|_{Y} \\
& \quad \leq K \varphi(x, x)+\frac{K^{2}}{48^{\beta}} \varphi(0,0)+\frac{K^{4}}{120^{\beta}}(\varphi(x, x)+\varphi(x,-x))+\frac{K^{4}}{720^{\beta}}(\varphi(2 x, 2 x)+\varphi(2 x,-2 x))
\end{aligned}
$$

for all $x \in X$. Multiply each side of (4.19) by $15^{\beta}$, we have

$$
\begin{aligned}
& \|15 f(4 x)-90 f(3 x)+240 f(2 x)-11190 f(x)\|_{Y} \\
& \quad \leq K 15^{\beta} \varphi(x, x)+\frac{K^{2} 5^{\beta}}{16^{\beta}} \varphi(0,0)+\frac{K^{4}}{8^{\beta}}(\varphi(x, x)+\varphi(x,-x))+\frac{K^{4}}{48^{\beta}}(\varphi(2 x, 2 x)+\varphi(2 x,-2 x))
\end{aligned}
$$

for all $x \in X$. By (4.15) and (4.20), we get

$$
\begin{aligned}
&\|20 f(3 x)-480 f(2 x)+16140 f(x)\|_{Y} \\
& \leq \frac{10^{\beta} K^{5}+K^{3}\left(225^{\beta}+36^{\beta}+1\right)}{720^{\beta}} \varphi(0,0)+\frac{K^{6}}{2^{\beta}} \varphi(0,2 x)+K^{4} \varphi(3 x, x) \\
&+K^{4} 6^{\beta} \varphi(2 x, x)+K^{2} 15^{\beta} \varphi(x, x) \\
&+\frac{K^{8}}{1440^{\beta}}(\varphi(6 x, 6 x)+\varphi(6 x,-6 x))+\frac{K^{9}}{240^{\beta}}(\varphi(4 x, 4 x)+\varphi(4 x,-4 x)) \\
&+\frac{K^{9}+K^{5} 2^{\beta}}{96^{\beta}}(\varphi(2 x, 2 x)+\varphi(2 x,-2 x))+\frac{K^{5}\left(15^{\beta}+1\right)}{120^{\beta}}(\varphi(x, x)+\varphi(x,-x))
\end{aligned}
$$


for all $x \in X$. By (4.18) and (4.21), we obtain

$$
\begin{aligned}
&\|360 f(2 x)-23040 f(x)\|_{Y} \\
& \leq \frac{10^{\beta} K^{6}+K^{4}\left(225^{\beta}+36^{\beta}+1\right)+K^{3} 200^{\beta}}{720^{\beta}} \varphi(0,0)+\frac{K^{7}}{2^{\beta}} \varphi(0,2 x)+K^{5} \varphi(3 x, x) \\
&+K^{5} 6^{\beta} \varphi(2 x, x)+K^{3} 15^{\beta} \varphi(x, x)+\frac{K^{9}}{1440^{\beta}}(\varphi(6 x, 6 x)+\varphi(6 x,-6 x)) \\
&+\frac{K^{10}}{240^{\beta}}(\varphi(4 x, 4 x)+\varphi(4 x,-4 x))+\frac{K^{6}}{12^{\beta}}(\varphi(3 x, 3 x)+\varphi(3 x,-3 x)) \\
&+\frac{K^{10}+K^{6}\left(2^{\beta}+8^{\beta}\right)}{96^{\beta}}(\varphi(2 x, 2 x)+\varphi(2 x,-2 x)) \\
&+\frac{K^{6}\left(15^{\beta}+1\right)+K^{5} 25^{\beta}}{120^{\beta}}(\varphi(x, x)+\varphi(x,-x))+K^{2} 10^{\beta} \varphi(0, x)
\end{aligned}
$$

for all $x \in X$. Therefore,

$$
\left\|f(2 x)-2^{6} f(x)\right\|_{Y} \leq \tilde{\varphi}(x)
$$

for all $x \in X$.

By Lemma 3.1, there exists a unique mapping $S: X \rightarrow Y$ such that $S(2 x)=2^{6} S(x)$ and

$$
\|f(x)-S(x)\|_{Y} \leq \frac{1}{64^{\beta}\left|1-L^{j}\right|} \tilde{\varphi}(x)
$$

for all $x \in X$. It remains to show that $S$ is a sextic map. By (4.2), we have

$$
\left\|\frac{D_{s} f\left(2^{j n} x, 2^{j n} y\right)}{64^{j n}}\right\|_{Y} \leq 64^{-j n \beta} \varphi\left(2^{j n} x, 2^{j n} y\right) \leq 64^{-j n \beta}\left(64^{j \beta} L\right)^{n} \varphi(x, y)=L^{n} \varphi(x, y)
$$

for all $x, y \in X$ and $n \in \mathbb{N}$. So

$$
\left\|D_{S} S(x, y)\right\|_{Y}=0
$$

for all $x, y \in X$. Thus the mapping $S: X \rightarrow Y$ is sextic, as desired.

Corollary 4.2. Let $X$ be a quasi- $\alpha$-normed space with quasi- $\alpha$-norm $\|\cdot\|_{X}$, and let $Y$ be a $(\beta, p)$ Banach space with $(\beta, p)$-norm $\|\cdot\|_{Y}$. Let $\delta, r$, s be positive numbers with $\lambda:=r+s \neq 6 \beta / \alpha$, and $f: X \rightarrow Y$ be a mapping satisfying

$$
\left\|D_{s} f(x, y)\right\|_{Y} \leq \delta\|x\|_{X}^{r}\|y\|_{X}^{s}
$$


for all $x, y \in X$. Then there exists a unique sextic mapping $S: X \rightarrow Y$ such that

$$
\|f(x)-S(x)\|_{Y} \leq \begin{cases}\frac{\delta \varepsilon_{r, s}}{64^{\beta}-2^{\alpha \lambda}}\|x\|_{X}^{\lambda}, & \lambda \in\left(0, \frac{6 \beta}{\alpha}\right), \\ \frac{2^{\lambda \alpha} \delta \varepsilon_{r, s}}{64^{\beta}\left(2^{\lambda \alpha}-64^{\beta}\right)}\|x\|_{X}^{\lambda}, & \lambda \in\left(\frac{6 \beta}{\alpha}, \infty\right),\end{cases}
$$

for all $x \in X$, where

$$
\begin{aligned}
\varepsilon_{r, s}:=\frac{K^{3}}{360^{\beta}}[ & K^{2} 3^{\alpha r}+K^{2} 6^{\beta} 2^{\alpha r}+15^{\beta}+\frac{2 \cdot K^{6} 6^{\alpha \lambda}}{1440^{\beta}}+\frac{2 \cdot K^{7} 4^{\alpha \lambda}}{240^{\beta}} \\
& \left.+\frac{2 \cdot K^{3} 3^{\alpha \lambda}}{12^{\beta}}+\frac{K^{3}\left(K^{4}+2^{\beta}+8^{\beta}\right) 2^{\alpha \lambda+1}}{96^{\beta}}+\frac{2 K^{2}\left(K 15^{\beta}+K+25^{\beta}\right)}{120^{\beta}}\right] .
\end{aligned}
$$

Corollary 4.3. Let $X$ be a quasi- $\alpha$-normed space with quasi- $\alpha$-norm $\|\cdot\|_{X}$, and let $Y$ be a $(\beta, p)$ Banach space with $(\beta, p)$-norm $\|\cdot\|_{Y}$. Let $\delta, \lambda$ be positive numbers with $\lambda \neq 6 \beta / \alpha$ and $f: X \rightarrow Y$ a mapping satisfying

$$
\left\|D_{s} f(x, y)\right\|_{Y} \leq \delta\left(\|x\|_{X}^{\lambda}+\|y\|_{X}^{\lambda}\right)
$$

for all $x, y \in X$. Then there exists a unique sextic mapping $S: X \rightarrow Y$ such that

$$
\|f(x)-S(x)\|_{Y} \leq \begin{cases}\frac{\delta \varepsilon_{\lambda}}{64^{\beta}-2^{\alpha \lambda}}\|x\|_{X^{\prime}}^{\lambda}, & \lambda \in\left(0, \frac{6 \beta}{\alpha}\right), \\ \frac{2^{\lambda \alpha} \delta \varepsilon_{\lambda}}{64^{\beta}\left(2^{\lambda \alpha}-64^{\beta}\right)}\|x\|_{X}^{\lambda}, & \lambda \in\left(\frac{6 \beta}{\alpha}, \infty\right),\end{cases}
$$

for all $x \in X$, where

$$
\begin{aligned}
\varepsilon_{\lambda}:=\frac{K^{2}}{360^{\beta}}[ & \frac{K^{5} 2^{\alpha \lambda}}{2^{\beta}}+K^{3}\left(3^{\alpha \lambda}+1\right)+K^{3} 6^{\beta}\left(2^{\alpha \lambda}+1\right)+2 \cdot K \cdot 15^{\beta}+\frac{4 \cdot K^{7} 6^{\alpha \lambda}}{1440^{\beta}}+\frac{4 \cdot K^{8} 4^{\alpha \lambda}}{240^{\beta}} \\
& \left.+\frac{4 \cdot K^{4} 3^{\alpha \lambda}}{12^{\beta}}+\frac{4 \cdot K^{4}\left(K^{4}+2^{\beta}+8^{\beta}\right) 2^{\alpha \lambda}}{96^{\beta}}+\frac{4 \cdot K^{3}\left(K \cdot 15^{\beta}+K+25^{\beta}\right)}{120^{\beta}}+10^{\beta}\right] .
\end{aligned}
$$

Corollary 4.4. Let $X$ be a quasi- $\alpha$-normed space with quasi- $\alpha$-norm $\|\cdot\|_{X}$, and let $Y$ be a $(\beta, p)$ Banach space with $(\beta, p)$-norm $\|\cdot\|_{\gamma}$. Let $\delta, r$, s be positive numbers with $\lambda:=r+s \neq 6 \beta / \alpha$ and $f: X \rightarrow Y$ a mapping satisfying

$$
\left\|D_{s} f(x, y)\right\|_{Y} \leq \delta\left[\|x\|_{X}^{r}\|y\|_{X}^{s}+\left(\|x\|_{X}^{r+s}+\|y\|_{X}^{r+s}\right)\right]
$$


for all $x, y \in X$. Then there exists a unique sextic mapping $Q: X \rightarrow Y$ such that

$$
\|f(x)-S(x)\|_{Y} \leq \begin{cases}\frac{\delta\left(\varepsilon_{r, s}+\varepsilon_{\curlywedge}\right)}{64^{\beta}-2^{\alpha \lambda}}\|x\|_{X}^{\lambda}, & \lambda \in\left(0, \frac{6 \beta}{\alpha}\right), \\ \frac{2^{\lambda \alpha} \delta\left(\varepsilon_{r, s}+\varepsilon_{\lambda}\right)}{64^{\beta}\left(2^{\lambda \alpha}-64^{\beta}\right)}\|x\|_{X^{\prime}}^{\lambda} & \lambda \in\left(\frac{6 \beta}{\alpha}, \infty\right),\end{cases}
$$

for all $x \in X$, where $\varepsilon_{r, s}$ and $\varepsilon_{\mathcal{\lambda}}$ are defined as in Corollaries 4.2 and 4.3 .

For the case $\lambda=6 \beta / \alpha$, similar to Example 3.6, we have the following counterexample. Example 4.5. Let $\phi: \mathbb{R} \rightarrow \mathbb{R}$ be defined by

$$
\phi(x)= \begin{cases}x^{6}, & \text { for }|x|<1 \\ 1, & \text { for }|x| \geq 1\end{cases}
$$

Consider that the function $f: \mathbb{R} \rightarrow \mathbb{R}$ is defined by

$$
f(x)=\sum_{n=0}^{\infty} 4^{-6 n} \phi\left(4^{n} x\right)
$$

for all $x \in \mathbb{R}$. Then $f$ satisfies the functional inequality

$$
\begin{aligned}
& \mid f(x+3 y)-6 f(x+2 y)+15 f(x+y)-20 f(x)+15 f(x-y) \\
& -6 f(x-2 y)+f(x-3 y)-720 f(y) \mid \\
& \quad \leq \frac{784 \cdot 4096^{3}}{4095}\left(x^{6}+y^{6}\right)
\end{aligned}
$$

for all $x, y \in \mathbb{R}$, but there do not exist a sextic mapping $S: \mathbb{R} \rightarrow \mathbb{R}$ and a constant $d>0$ such that $|f(x)-S(x)| \leq d \quad x^{6}$ for all $x \in \mathbb{R}$.

Proof. It is clear that $f$ is bounded by $4096 / 4095$ on $\mathbb{R}$. If $x^{6}+y^{6}=0$ or $x^{6}+y^{6} \geq 1 / 4096$, then

$$
\left|D_{s} f(x, y)\right| \leq \frac{784 \cdot 4096}{4095} \leq \frac{784 \cdot 4096^{2}}{4095}\left(x^{6}+y^{6}\right)
$$

Now suppose that $0<x^{6}+y^{6}<1 / 4096$. Then there exists a non-negative integer $k$ such that

$$
\frac{1}{4096^{k+2}} \leq x^{6}+y^{6}<\frac{1}{4096^{k+1}} .
$$


Similar to the proof of Example 3.6 we obtain that

$$
\left|D_{s} f(x, y)\right| \leq \frac{784 \cdot 4096^{3}}{4095}\left(x^{6}+y^{6}\right)
$$

Therefore, $f$ satisfies (4.37) for all $x, y \in \mathbb{R}$. Now, we claim that the functional equation (1.5) is not stable for $\lambda=6$ in Corollary $4.3(\alpha=\beta=p=1)$. Suppose on the contrary that there exists a sextic mapping $S: \mathbb{R} \rightarrow \mathbb{R}$ and constant $d>0$ such that $|f(x)-S(x)| \leq d x^{6}$ for all $x \in \mathbb{R}$. Then there exists a constant $c \in \mathbb{R}$ such that $S(x)=c x^{6}$ for all rational numbers $x$ (see [7]). So we obtain that

$$
|f(x)| \leq(d+|c|) x^{6}
$$

for all $x \in \mathbb{Q}$. Let $m \in \mathbb{N}$ with $m+1>d+|c|$. If $x$ is a rational number in $\left(0,4^{-m}\right)$, then $4^{n} x \in(0,1)$ for all $n=0,1, \ldots, m$, and for this $x$ we get

$$
f(x)=\sum_{n=0}^{\infty} \frac{\phi\left(4^{n} x\right)}{4^{6 n}} \geq \sum_{n=0}^{m} \frac{\left(4^{n} x\right)^{6}}{4^{6 n}}=(m+1) x^{6}>(d+|c|) x^{6},
$$

which contradicts (4.41).

\section{Acknowledgment}

The first author was supported by the National Natural Science Foundation of China (10671013, 60972089).

\section{References}

[1] Z. Moszner, "On the stability of functional equations," Aequationes Mathematicae, vol. 77, no. 1-2, pp. 33-88, 2009.

[2] S. M. Ulam, A Collection of Mathematical Problems, Interscience Tracts in Pure and Applied Mathematics, Interscience Publishers, New York, NY, USA, 1960.

[3] D. H. Hyers, "On the stability of the linear functional equation," Proceedings of the National Academy of Sciences of the United States of America, vol. 27, pp. 222-224, 1941.

[4] T. M. Rassias, "On the stability of the linear mapping in Banach spaces," Proceedings of the American Mathematical Society, vol. 72, no. 2, pp. 297-300, 1978.

[5] P. Găvruţa, "A generalization of the Hyers-Ulam-Rassias stability of approximately additive mappings," Journal of Mathematical Analysis and Applications, vol. 184, no. 3, pp. 431-436, 1994.

[6] Z. Gajda, "On stability of additive mappings," International Journal of Mathematics and Mathematical Sciences, vol. 14, no. 3, pp. 431-434, 1991.

[7] K.-W. Jun and H.-M. Kim, "On the stability of Euler-Lagrange type cubic mappings in quasi-Banach spaces," Journal of Mathematical Analysis and Applications, vol. 332, no. 2, pp. 1335-1350, 2007.

[8] T. Z. Xu, J. M. Rassias, and W. X. Xu, "A fixed point approach to the stability of a general mixed AQCQ-functional equation in non-archimedean normed spaces," Discrete Dynamics in Nature and Society, vol. 2010, Article ID 812545, 24 pages, 2010.

[9] D. Miheț and V. Radu, "On the stability of the additive Cauchy functional equation in random normed spaces," Journal of Mathematical Analysis and Applications, vol. 343, no. 1, pp. 567-572, 2008.

[10] C. Park, "Fixed points and the stability of an AQCQ-functional equation in non-Archimedean normed spaces," Abstract and Applied Analysis, vol. 2010, Article ID 849543, 15 pages, 2010. 
[11] J. Sikorska, “On a direct method for proving the Hyers-Ulam stability of functional equations," Journal of Mathematical Analysis and Applications, vol. 372, no. 1, pp. 99-109, 2010.

[12] J. Brzdęk, "On a method of proving the Hyers-Ulam stability of functional equations on restricted domains," The Australian Journal of Mathematical Analysis and Applications, vol. 6, no. 1, article 4, p. 10, 2009.

[13] G.-L. Forti, "Comments on the core of the direct method for proving Hyers-Ulam stability of functional equations," Journal of Mathematical Analysis and Applications, vol. 295, no. 1, pp. 127-133, 2004.

[14] G.-L. Forti, "Elementary remarks on Ulam-Hyers stability of linear functional equations," Journal of Mathematical Analysis and Applications, vol. 328, no. 1, pp. 109-118, 2007.

[15] K. Ravi, M. Arunkumar, and J. M. Rassias, "Ulam stability for the orthogonally general EulerLagrange type functional equation," International Journal of Mathematics and Statistics, vol. 3, no. A08, pp. 36-46, 2008.

[16] T. Z. Xu, J. M. Rassias, and W. X. Xu, "Stability of a general mixed additive-cubic functional equation in non-Archimedean fuzzy normed spaces," Journal of Mathematical Physics, vol. 51, no. 6, Article ID 093508, 19 pages, 2010.

[17] T. Z. Xu, J. M. Rassias, and W. X. Xu, "A fixed point approach to the stability of a general mixed additive-cubic functional equation in quasi fuzzy normed spaces," International Journal of Physical Sciences, vol. 6, no. 2, 12 pages, 2011.

[18] T. Z. Xu, J. M. Rassias, and W. X. Xu, “Intuitionistic fuzzy stability of a general mixed additive-cubic equation," Journal of Mathematical Physics, vol. 51, no. 6, Article ID 063519, 21 pages, 2010.

[19] T. Z. Xu, J. M. Rassias, and W. X. Xu, "On the stability of a general mixed additive-cubic functional equation in random normed spaces," Journal of Inequalities and Applications, vol. 2010, Article ID 328473, 16 pages, 2010.

[20] M. Mohamadi, Y. J. Cho, C. Park, P. Vetro, and R. Saadati, "Random stability of an additive-quadratic quartic functional equation," Journal of Inequalities and Applications, vol. 2010, Article ID 754210, 18 pages, 2010.

[21] E. Baktash, Y. J. Cho, M. Jalili, R. Saadati, and S. M. Vaezpour, “On the stability of cubic mappings and quadratic mappings in random normed spaces," Journal of Inequalities and Applications, vol. 2008, Article ID 902187, 11 pages, 2008.

[22] L. Cădariu and V. Radu, "Fixed points and stability for functional equations in probabilistic metric and random normed spaces," Fixed Point Theory and Applications, vol. 2009, Article ID 589143, 18 pages, 2009.

[23] M. Eshaghi Gordji and M. B. Savadkouhi, "Stability of mixed type cubic and quartic functional equations in random normed spaces," Journal of Inequalities and Applications, vol. 2009, Article ID 527462, 9 pages, 2009.

[24] J. M. Rassias and H.-M. Kim, "Generalized Hyers-Ulam stability for general additive functional equations in quasi- $\beta$-normed spaces," Journal of Mathematical Analysis and Applications, vol. 356, no. 1, pp. 302-309, 2009.

[25] J. M. Rassias, "Solution of the Ulam stability problem for cubic mappings," Glasnik Matematički. Serija III, vol. 36, no. 1, pp. 63-72, 2001.

[26] J. M. Rassias, "Solution of the Ulam stability problem for quartic mappings," Glasnik Matematički. Serija III, vol. 34, no. 2, pp. 243-252, 1999.

[27] R. Saadati, S. M. Vaezpour, and Y. J. Cho, “On the stability of cubic mappings and quartic mappings in random normed spaces," Journal of Inequalities and Applications, vol. 2009, Article ID 214530, 6 pages, 2009.

[28] S.-M. Jung and J. Brzdęk, "A note on stability of a linear functional equation of second order connected with the Fibonacci numbers and Lucas sequences," Journal of Inequalities and Applications, vol. 2010, Article ID 793947, 10 pages, 2010.

[29] T. Z. Xu, J. M. Rassias, and W. X. Xu, "A generalized mixed quadratic-quartic functional equation," to appear in Bulletin of the Malaysian Mathematical Sciences Society.

[30] G. Isac and T. M. Rassias, "Stability of $\psi$-additive mappings: applications to nonlinear analysis," International Journal of Mathematics and Mathematical Sciences, vol. 19, no. 2, pp. 219-228, 1996. 